\title{
ON NORMAL COMPLEMENTS TO SECTIONS OF FINITE GROUPS
}

\author{
EVERETT C. DADE \\ (Received 2 January 1973) \\ Communicated by G. E. Wall
}

Suppose that $H / N$ is a section of a finite group $G$, i.e., that $H$ is a subgroup of $G$ and $N$ is a normal subgroup of $H$. We are interested in the existence of normal subgroups $M$ of $G$ satisfying:

$$
\begin{gathered}
M \cap H=N, \\
M H=G .
\end{gathered}
$$

Such an $M$ can be called a normal complement to the section $H / N$ in $G$.

Of course, one must add further conditions in order to guarantee the existence of $M$. Since (0.1) implies that inclusion induces an isomorphism of $H / N$ onto $G / M$, one obviously necessary condition is:

$$
\begin{gathered}
\text { Any elements } \sigma, \tau \in H \text { which are conjugate in } G \text { have } \\
\text { images } \sigma N, \tau N \text { which are conjugate in } H / N .
\end{gathered}
$$

Just as obviously, this condition is not sufficient (let $G$ be a non-abelian nilpotent group, $H$ be its center, and $N$ be 1).

The question of what must be added to (0.2) has intrigued several authors over the yeras, especially Brauer (1964). Recently Friesen (1974) showed that (0.2) implied the existence of $M$ whenever $G$ was solvable and $H$ was a $\pi$-Hall subgroup of $G$, for some set of primes $\pi$. Since the Hall subgroups of solvable groups have all the nice properties one could wish, Friesen's result suggests the following question: How far down the list $E_{\pi}, C_{\pi}, D_{\pi}$ (see Hall (1956)) of increasingly stringent conditions for a $\pi$-Hall sungroup $H$ of an arbitrary finite group $G$ must one go before (0.2) implies the existence of $M$ ? Friesen's example (in section 1 of Friesen (1974)), in which $G$ is the symmetric group $\Sigma_{p}$ on a prime number $p \geqq 5$ of letters, $H$ is its Hall $p^{\prime}$-subgroup $\Sigma_{p-1}$, and $N$ is 1 , shows that $C_{\pi}$ (the conjugacy of all Hall $\pi$-subgroups of $G$ ) does not suffice. However, we shall 
show that the next condition $D_{\pi}$ is sufficient, i.e., that $M$ exists whenever $(0.2)$ holds and $H$ is a Hall $\pi$-subgroup of $G$ containing at least one conjugate of every $\pi$-subgroup $E$ of $G$.

Our actual result has even weaker hypotheses. When you know that one of the key tools in its proof is Brauer's characterization of group characters, it is not surprising that the condition $D_{\pi}$ can be weakened so as only to require that $H$ contain at least one conjugate of every Brauer elementary $\pi$-subgroup $E$ of $G$, i.e., of those $\pi$-subgroups $E$ which are direct products of cyclic groups with $p$-groups (the "elementary" subgroups of Brauer and Tate (1965) and Brauer (1964)). We can also (following (AI) of Brauer (1964)) replace the condition that $H$ be a $\pi$-Hall subgroup of $G$ by the weaker hypothesis that $H / N$ be a $\pi$-group and that $[G: H]$ be a $\pi^{\prime}$-number (i.e., be divisible only by promes not in $\pi$ ). Since the Brauer elementary $\pi$-subgroups $E$ of $G$ include the $p$-Sylow subgroups, for every prime $p \in \pi$, this weaker hypothesis is a consequence of the above one (we owe this remark to a friendly letter of $\mathbf{M}$. Isaacs). So our theorem is:

THEOREM 1. Suppose that $H / N$ is a $\pi$-section of a finite group $G$ satisfying (0.2) and that:

$$
\begin{gathered}
\text { Any Brauer elementary } \pi \text {-subgroup } E \text { of } G \text { is } \\
\text { G-conjugate to a subgroup of } H .
\end{gathered}
$$

Then $H / N$ has a unique normal complement $M$ in $G$.

The special case $N=1$ of this theorem was proved by Brauer as Theorem 3 of Brauer (1964). Brauer obtained this case as a consequence of a more complicated result (Theorem 1 of Brauer (1964)). The methods we use to prove our Theorem 1 can also be used to improve Brauer's Theorem 1 by showing that his annoying hypothesis (AIV) (see section 6 of Brauer (1964)) is actually a cobsequence of his other hypotheses (AI-III). After another minor improvement in his hypothesis (AIII), his theorem becomes:

TheOREM 2. (Brauer, improved). Suppose that $H / N$ is a $\pi$-section of a finite group $\mathrm{G}$ satisfying $(0.2)$ and that:

$$
[G: H] \text { is a } \pi^{\prime} \text {-number, }
$$

(0.4b) If $\sigma$ is a $\pi$-element of $H-N$ and if $P$ is a p-Sylow subgroup of the centralizer $C_{\mathrm{G}}(\sigma)$ of $\sigma$ in $G$, for some prime $p \in \pi$ not dividing the order of $\sigma$, then the sub-

$$
\text { group }\langle\sigma\rangle \times P \text { is G-conjugate to a subgroup of } H \text {. }
$$

Then $H / N$ has a unique normal complement $M$ in $G$.

Of course, (0.4) is weaker than (0.3). So Theorem 2 implies Theorem 1. 
Nevertheless, Theorem 1 is so easy to prove directly that it seems better to do so in section 1, while in section 2 we content ourselves with indicating the modifications necessary in Brauer's proof to obtain Theorem 2.

It would be nice to be able to weaken the hypothesis ( $0.4 \mathrm{a})$, but this seems to be very difficult.

\section{Proof of Theorem 1}

As for a long line of theorems going back to Frobenius' construction of normal complements in Frobenius groups, the proof of Theorem 1 is based on the observation that one can construct the irreducible characters of the factor group $G / M$ (considered as characters of $G$ ) without knowing that $M$ exists. Then $M$ is obtained as the intersection of the kernels of those characters.

To see how this can be done, suppose for a moment that $M$ exists. Let $\eta: H \rightarrow H / N$ and $\eta^{*}: G \rightarrow H / N(\simeq G / M)$ be the natural epimorphisms. Then the conjugacy classes $k_{1}=\{1\}, k_{2}, \cdots, k_{n}$ of $H / N$ have inverse images $K_{i}=\eta^{-1}\left(k_{i}\right)$, $K_{i}^{*}=\left(\eta^{*}\right)^{-1}\left(k_{i}\right)$ in $H, G$, respectively, for $i=1, \cdots, n$. Evidently $(0.1)$ implies:

$$
K_{i}^{*} \cap H=K_{i}, \text { for } i=1, \cdots, n .
$$

Because $G / M \simeq H / N$ is a $\pi$-group, an element $\sigma \in G$ lies in some $K_{i}^{*}$ if and only if its $\pi$-part $\sigma_{\pi}$ (in the usual sense of the term as in Brauer (1964)) lies in $K_{i}^{*}$. By $(0.3)$ the $\pi$-element $\sigma_{n}$ of $G$ is $G$-conjugate to an element $\tau \in H$. Since $K_{i}^{*}$ is closed under $G$-conjugation, it follows from (1.1) that $\sigma \in K_{i}^{*}$ if and only if $\tau \in K_{i}^{*} \cap H=K_{i}$. So $K_{i}^{*}$ is given by:

$$
\begin{gathered}
K_{i}^{*}=\left\{\sigma \in G \mid \sigma_{n} \text { is } G \text {-conjugate to an element of } K_{i}\right\}, \\
\text { for } i=1, \cdots, n .
\end{gathered}
$$

This is a description of the set $K_{i}^{*}$ which does not depend upon $M$. In particular, it shows that $M=K_{1}^{*}$ is unique if it exists.

Let $\phi_{1}=1, \phi_{2}, \cdots, \phi_{n}$ be the complex irreducible characters of $H / N$. If we choose a representative $\rho_{i}$ in each class $k_{i}$ of $H / N$, then the corresponding irreducible characters $\Phi_{j}=\phi_{j} \circ \eta$ and $\Phi_{j}^{*}=\phi_{j} \circ \eta^{*}$ of $H$ and $G$, respectively, are given by:

$$
\begin{aligned}
& \Phi_{j}(\tau)=\phi_{j}\left(\rho_{i}\right), \text { for all } \tau \in K_{i}, i, j=1, \cdots, n, \\
& \Phi_{j}^{*}(\sigma)=\phi_{j}\left(\phi_{i}\right), \text { for all } \sigma \in K_{i}^{*}, i, j=1, \cdots, n .
\end{aligned}
$$

In view of (1.2), this gives us the desired description of the irreducible characters $\Phi_{1}^{*}, \cdots, \Phi_{n}^{*}$ of $G / M$ without reference to $M$.

Now we start from the hypotheses of Theorem 1 without supposing the existence of $M$. Since we do have the epimorphism $\eta: H \rightarrow H / N$, we can define the $k_{i}, K_{i}, \phi_{j}$ and $\Phi_{j}$ as above. We now use (1.2) as the definition of the subset $K_{i}^{*}$ of $G$, for $i=1, \cdots, n$. Hypothesis (0.2) implies that any $\pi$-element of $K_{i}^{*} \cap H$ 
lies in $K_{i}$. Because $H / N$ is a $\pi$-group, an element $\tau \in H$ lies in $K_{i}$ if and only if its $\pi$-part $\tau_{\pi}$ lies in $K_{i}$. It follows that (1.1) holds in the present situation $\alpha$

By $(0.3)$ every $\pi$-element of $G$ is conjugate to an element of $H$, and hence to an element of some $K_{i}$. This and (1.2) tell us that $G$ is the union of the $K_{i}^{*}$. Since the $K_{i}$ are pairwise disjoint, it follows from (1.1) and (1.2) that the $K_{i}^{*}$ are pairwise disjoint. Hence $G$ is the disjoint union:

$$
G=K_{1}^{*} \dot{\cup} K_{2}^{*} \dot{u} \ldots \dot{\cup} K_{n}^{*} \text {. }
$$

In view of (1.4) we can use (1.3b) to define the class functfions $\Phi_{j}^{*}$ on $G$. From (1.1), (1.2), and (1.3a) we conclude that:

$$
\begin{gathered}
\Phi_{j}^{*}(\sigma)=\Phi_{j}^{*}\left(\sigma_{\pi}\right), \text { for all } \sigma \in G, j=1, \cdots, n . \\
\left.\Phi_{j}^{*}\right|_{H}=\Phi_{j}, \text { for all } j=1, \cdots, n .
\end{gathered}
$$

Next we must show:

$$
\begin{gathered}
\text { Each } \Phi_{j}^{*}, j=1, \cdots, n, \text { is a generalized character of } \\
G(i . e ., \text { an integral linear combination of the complex } \\
\text { irreducible characters of } G) .
\end{gathered}
$$

Since $\Phi_{j}^{*}$ is a complex class function on $G$, it suffices by a well-known theorem of Brauer (see Brauer and Tate (1965)) to show that the restriction $\left.\Phi_{j}^{*}\right|_{\mathbf{E}}$ is a generalized character of $E$ for any Brauer elementary subgroup $E$ of $G$. The group $E$, being nilpotent, is the direct product $E=E_{\pi} \times E_{\pi^{\prime}}$ of its Hall $\pi$-and $\pi^{\prime}$-subgroups $E_{\pi^{\prime}}$ and $E_{\pi^{\prime}}$ (respectively), both of which are also Brauer elementary. By (1.5a) the restriction $\left.\Phi_{j}^{*}\right|_{E}$ is just the composition of the restrication $\left.\Phi_{j}^{*}\right|_{E_{\pi}}$ with the projection of $E$ onto $E_{\pi}$. So we only need show that the latter restriction is a generalized character of $E_{\pi}$, i.e., we may assume that $E=E_{\pi}$ is a Brauer elementary $\pi$-subgroup of $G$. Now (0.3) tells us that $E$ is $G$-conjugate to a subgroup of $H$. Since $\Phi_{j}^{*}$ is a class function on $G$, we may replace $E$ by its conjugate and suppose that $E \leqq H$. But then $\left.\Phi_{j}^{*}\right|_{E}=\left.\Phi_{j}\right|_{E}$ by $(1.5 \mathrm{~b})$, and $\Phi_{j}$ is a character of $H$ by construction. Hence $\left.\Phi_{j}^{*}\right|_{E}$ is a character of $E$ and the proof of (1.6) is complete.

For each $i=1, \cdots, n$, we form the class function:

$$
\Psi_{i}=\sum_{j=1}^{n} \phi_{j}\left(\rho_{i}^{-1}\right) \Phi_{j}^{*}
$$

on $G$. In view of (1.3b) and the orthogonality relations for the irreducible characters $\phi_{j}$ of $H / N$, the values of $\Psi_{i}$ are given by:

It follows that:

$$
\begin{aligned}
\Psi_{i} & =\left|C_{H / N}\left(\rho_{i}\right)\right| & \text { on } & K_{i}^{*} \\
& =0 & \text { on } & G-K_{i}^{*} .
\end{aligned}
$$


Evidently

$$
\left(\Psi_{i}, 1\right)_{\mathrm{G}}=\frac{1}{|G|} \sum_{\sigma \in \mathrm{G}} \Psi_{i}(\sigma)=\frac{\left|C_{H / N}\left(\rho_{i}\right)\right| \cdot\left|K_{i}^{*}\right|}{|G|} .
$$

$$
\frac{|G|}{\left|C_{H / N}\left(\rho_{i}\right)\right|}=[G: H] \cdot|N| \cdot\left[H / N: C_{H / N}\left(\rho_{\iota}\right)\right]=[G: H] \cdot|N| \cdot\left|k_{i}\right| \cdot
$$

So the above inner product can be written as:

$$
\left(\Psi_{i}, 1\right)_{G}=\frac{\left|K_{i}^{*}\right|}{[G: H] \cdot|N| \cdot\left|k_{i}\right|} .
$$

From (1.6) and (1.7) it is clear that $\Psi_{i}$ is a linear combination of the irreducible complex characters of $G$ with algebraic integers as coefficients. Hence the coefficient $\left(\Psi_{i}, 1\right)_{G}$ of 1 is an algebraic integer. By $(1.8)$ it is also a rational number. Therefore it is an ordinary integer, and (1.8) says that $[G: H] \cdot|N| \cdot\left|k_{i}\right|$ divides $\left|K_{i}^{*}\right|$. Because $K_{i}^{*}$ is nonempty (by (1.1)), this implies:

$$
[G: H] \cdot|N| \cdot\left|k_{i}\right| \leqq\left|K_{i}^{*}\right|, \text { for } i=1, \cdots, n .
$$

Adding the inequalities (1.9) we obtain:

$$
[G: H] \cdot|N| \cdot \sum_{i=1}^{n}\left|k_{i}\right| \leqq \sum_{i=1}^{n}\left|K_{i}^{*}\right| .
$$

But $k_{1}, \cdots, k_{n}$ are the conjugacy classes of $H / N$. so $\sum_{i=1}^{n}\left|k_{i}\right|=|H / N|$, and the left side of $(1.10)$ is $[G: H] \cdot|N| \cdot|H / N|=|G|$. The right side is also $|G|$ by (1.4). Therefore (1.10) is equality, which implies that (1.9) is equality for all $i$ :

$$
\left|K_{i}^{*}\right|=[G: H] \cdot|N| \cdot\left|k_{i}\right|, \text { for } i=1, \cdots, n .
$$

We use this, (1.4), and (1.3b) to compute:

$$
\begin{aligned}
\left(\Phi_{j}^{*}, \Phi_{j}^{*}\right)_{G} & =\frac{1}{|G|} \sum_{\sigma \in G}\left|\Phi_{j}^{*}(\sigma)\right|^{2}=\frac{1}{|G|} \sum_{i=1}^{n}\left|K_{i}^{*}\right|\left|\phi_{i}\left(\rho_{i}\right)\right|^{2} \\
& =\frac{[G: H] \cdot|N|}{|G|} \sum_{i=1}^{n}\left|k_{i}\right|\left|\phi_{j}\left(\rho_{i}\right)\right|^{2}=\frac{1}{|H / N|} \sum_{\rho \in H / N}\left|\phi_{j}(\rho)\right|^{2} \\
& =\left(\phi_{j}, \phi_{j}\right)_{H / N}=1 .
\end{aligned}
$$

Because $\Phi_{j}^{*}(1)=\phi_{j}(1)>0$, this and (1.6) imply that $\Phi_{j}^{*}$ is an irreducible complex character of $G$. Hence its kernel:

$$
\operatorname{Ker}\left(\Phi_{j}^{*}\right)=\left\{\sigma \in G \mid \Phi_{j}^{*}(\sigma)=\Phi_{j}^{*}(1)\right\}
$$

is a normal subgroup of $G$. By (1.3b) and the fact that $\cap_{j=1}^{n} \operatorname{Ker}\left(\phi_{j}\right)=\{1\}=k_{1}$, the intersection $\cap_{j=1}^{n} \operatorname{Ker}\left(\Phi_{j}^{*}\right)$ is precisely $K_{1}^{*}$. Hence $M=K_{1}^{*}$ is a normal subgroup of $G$ satisfying (0.1a) by (1.1) (since $N=\eta^{-1}(1)=K_{1}$ ). Furthermore, 


$$
|M|=\left|K_{1}^{*}\right|=[G: H] \cdot|N|
$$

by (1.11), so that:

$$
|H M|=\frac{|H| \cdot|M|}{|H \cap M|}=\frac{|H| \cdot[G: H] \cdot|N|}{|N|}=|G| .
$$

Therefore $M$ also satisfies $(0.1 \mathrm{~b})$, and the theorem is proved.

\section{Proof of Theorem 2}

We simply indicate the modifications necessary in the arguments of sections 4 and 5 of Brauer (1964) in order to change Brauer's proof of his Theorem 1 into a proof of our Theorem 2. Lemmas 1-5 in section 4 of Brauer (1964) are consequences of Brauer's axioms (AI-III) alone. His axioms (AI) and (AII) are our (0.4a) and (0.2), respectively. His axiom (AIII) is used only twice, in the proofs of his Lemmas 2 and 4 . In each case it can obviously be replaced by our $(0.4 \mathrm{~b})$ without changing his arguments. So all the results of section 4 of Brauer (1964) hold in our situation. In particular, if $K_{i}^{*}$ is defined by (1.2), for $i>1$, while $K_{1}^{*}$ is defined to be $G-\cap_{i=2}^{n} K_{i}^{*}$, then our (1.1) and (1.4) hold in virtue of Lemma 1 of Brauer (1964).

As before, we define the class functions $\Phi_{j}^{*}$ by (1.3b). Brauer's argument showing that (1.6) holds, as given on pages 76 and 77 of Brauer (1964) depends only on his axiom (AI)(which is our (0.4a)) and the results of his section 4. So it is still valid. Now the rest of the proof of Theorem 2 can be completed by repeating the argument of section 1 above, starting at (1.7).

Notice that the inequalities (1.9), which are obtained in the course of this last argument, imply that Brauer's axiom (AIV) holds. Thus (AIV) is actually a consequence of (AI-III), as remarked earlier.

\section{References}

R. Brauer (1964), 'On quorient groups of finite groups', Math. Zeitschrift 83 72-84.

R. Brauer and J. Tate (1955), 'On the characters of finite groups', Ann. Math. 62, 1-7.

D. Friesen (1974), 'Normal complements in finite solvable groups', J. Austral. Math. Soc. 18, 212-214.

P. Hall (1956), ‘Theorems like Sylow's', Proc. London Math. Soc. (3) 6, 286-304.

Mathematics Department

University of Illinois

Urbana, Illinois 61801

U. S. A. 